\section{NP18 (continued)}

transdisciplinary coursework, experiential learning, and research focused on childhood obesity prevention. To date, 50 students from seven disciplines have completed the certificate. Perceptions of program impact were assessed from 2012 through 2016, via: focus groups, course evaluations, exit interviews, and alumni surveys. Focus groups and course evaluations were completed after students finished TOP core courses. Exit interviews were completed annually within six months of program completion and alumni surveys were completed in 2016 with alumni who completed the certificate between 2012-2016.

Evaluation: Substantial gains in confidence were observed in students' abilities to make knowledgeable decisions to prevent childhood obesity, recommend practices related to transdisciplinary approaches, think critically about childhood obesity prevention, and understand perspectives of other disciplines. The majority of alumni reported that the program helped prepare them for their current professional positions; specifically preparing them for working collaboratively with transdisciplinary teams, and with research and writing skills. Ninety-eight percent of alumni reported they would recommend the TOP program to other students, noting improved interprofessional teamwork and collaboration skills.

Conclusion and Implications: Program evaluations demonstrate the positive impact this certificate program plays in developing graduates from across disciplines to be effective collaborators in tackling obesity prevention measures. The TOP certificate program serves as a premier example for development of transdisciplinary academic programs created to prepare future professionals to solve real-life problems.

Funding: 2011-67002-30202

\section{NP19 Mobilizing Rural Low-Income Communities to Assess and Improve the Ecological Environment to Prevent Childhood Obesity}

Paula Peters,PhD, ppeters@ksu.edu, Kansas State University, 1324 Lovers Lane, 340 Justin Hall, Manhattan, KS 66502; Amy Mobley, PhD, RD, University of Connecticut; Sandy Procter, $P h D, R D, L D$, Kansas State University; Carol Smathers, MPH, MS, The Ohio State University; Dawn Contreras, PhD, Univeristy of Michigan; Abby Gold, PhD, MPH, RD, North Dakota State University; Renee Oscarson, $P h D, R D, L N, B C C$, South Dakota State University; Ann Keim, PhD, University of Wisconsin

Objective: To mobilize capacity, including policy, system and environmental changes in rural communities to create and sustain an environment and culture of healthful eating and physical activity to prevent childhood obesity. Description: A collaborative multidisciplinary team from Indiana, Kansas, Michigan, North Dakota, Ohio, South Dakota, and Wisconsin implemented a community development model testing the value of a community coach in assisting existing health coalitions in improving community environments. Two closely matched communities in each state (randomly assigned as either intervention with a hired coach or comparison with no coach) were provided with funding to support the promotion of healthy eating and increased physical activity of four-year-olds in their community. Additionally, intervention communities received assistance from a community coach. Community environments were examined before and after four years of implementation. Training on the methods of community coaching was provided to the comparison communities at the end of the project.

Evaluation: Enhancement of a community's capacity to prevent childhood obesity with community coaching has been and continues to be examined through a mixed methods data analysis approach. Assessments used previously validated instruments, reflections, coalition assessments, interviews and a final ripple mapping exercise.

Conclusions and Implications: Introduction and successful use of a new community-based coaching model benefited the intervention communities and they were able to address more policy, system and environmental approaches. Best practices were identified and an online tutorial developed. Sharing of results will be ongoing.

Funding: 2011-68001-30100

\section{NP20 Supermarket Science: Multipronged Approaches to Increasing Fresh, Frozen, and Canned Fruit \& Vegetable Purchases}

Michele Polacsek, PhD, MHS, mpolacsek@une.edu, University of New England, 716 Stevens Avenue, Portland, ME 04102; Alyssa Moran, MPH, RD, Hard T.H. Chan School of Public Health; Anne Thorndike, MPH, MD, Harvard Medical School; Rebecca Boulos, PhD, MPH, University of Southern Maine; Rebecca Franckle, MPH, ScD; Julie Greene, BS, Hannaford Brothers Supermarkets; Daniel Blue, BS; Jason Block, MPH, MD, Harvard Medical School; Eric Rimm, ScD, Harvard T.H. Chan School of Public Health

Objective: Conduct a randomized controlled study to determine if a supermarket double-dollar fruit and vegetable (FV) incentive program increases FV purchases among low income families.

Description: We enrolled 401 customers from a supermarket in a low-income rural community. Purchases were tracked using a loyalty card system that provided study participants with a 5\% discount on all purchases during a 3-month baseline period followed by the four-month intervention. The intervention group received half-off fresh, frozen or canned FV; the control group received no incentives. Primary outcomes were changes in weekly spending on FV between baseline and intervention periods. Secondary analyses examined changes among SNAP-eligible participants.

Evaluation: Weekly spending on total FV increased in the intervention arm, compared to control (15\% increase; $\$ 1.83,95 \% \mathrm{CI}=\$ 0.28-3.84)$. The largest increase was for fresh FV (18\% increase: $\$ 1.97,95 \% \mathrm{CI}=\$ 0.49-3.44)$ with

Continued on page $S 118$ 


\section{NP20 (continued)}

little change for canned and frozen FV. Secondary analyses revealed even greater increases in weekly FV spending among SNAP-eligible participants compared to non-SNAP-eligible participants (45\% increase; $\$ 2.37$, $95 \% \mathrm{CI}=\$-0.04-4.78)$ compared to $(11 \%$ increase; $\$ 1.57,95 \% \mathrm{CI}=\$-0.36-3.50)$, and increased fresh $\mathrm{FV}$ spending among SNAP-eligible participants $(53 \%$ increase; $\$ 2.55,95 \% \mathrm{CI}=\$ 0.23-4.87)$ compared to nonSNAP eligible participants (13\% increase; $\$ 1.68$, 95\% $\mathrm{CI}=\$-0.15-3.52)$.

Conclusions and Implications: A double-dollar pricing incentive increased FV supermarket purchases in a low-income community. SNAP-eligible customers had the greatest improvements. These results suggest that financial incentives could be an effective strategy for the SNAP program to improve healthy food choices.

Funding: 2016-68001-24961

\section{NP21 Latino Fathers Promoting Healthy Youth Behaviors}

Ghaffar Ali Hurtado, PhD, University of Maryland; Youjie Zhang, MS, University of Minnesota; Patty ArellanoBrazys, BS; Alejandro Reyes-Peralta, BS, RD;

Marla Reicks, PhD, RD, mreicks@umn.edu, 1334 Eckles

Avenue, Saint Paul, MN 55108

Objective: Padres Preparados, Ovenes Saludables is an eight-session intervention program for Latino parents, especially fathers, and youth (10-14 years) to improve parenting practices and youth energy balance-related behaviors. University of Minnesota researchers and Latinoserving organizations are collaborating to develop, implement and evaluate the effectiveness of the program in Minneapolis/St. Paul and rural Minnesota over five years. Description: An existing parenting skills program is being revised with community partners based on focus group interview results with parents and children $(n=60)$. Parenting skills addressed by the program include parenting styles, communication, discipline/control, supervision, and connection. Focus groups explored parenting practices and youth perspectives on parental supports and cultural traditions. Transcripts were coded and sorted using NVivo software and read for common themes. Findings identified: specific youth and parent behaviors and parenting practices to address (role modeling healthy behaviors, setting healthful expectations, creating a supportive environment), barriers and facilitators for behaviors and parenting practices, and primary considerations for effective community-based programs. Discussions among a father advisory board ( $n=6$ fathers) were held in four 90 minute sessions regarding program priorities and characteristics to increase relevance among fathers.

Evaluation: A randomized-controlled trial $(\mathrm{n}=240$ youth, 240 mothers, 240 fathers) is planned for years 24 with assessments pre-, immediate post-, and 3 months post-course. Outcomes include youth fruit and vegetable, sugar sweetened beverage, snacks, sweets, fast food and family meal intake, physical activity and screen time.
Conclusions and Implications: Formative findings are being used to develop and pilot-test the intervention format, materials, and activities to design a culturally-relevant, effective intervention.

Funding: 2016-68001-24921

\section{NP22 Cooking Matters for Families Revised to Improve Family Vegetable Outcomes}

Francine Overcash, MPH, University of Minnesota; Allison Ritter, MS; Zata Vickers, PhD; Marla Reicks, PhD, RD, mreicks@umn.edu, 1334 Eckles Avenue, Saint Paul, MN 55108

Objective: A controlled-trial is being conducted to determine the effectiveness of Cooking Matters for Families ${ }^{\mathrm{TM}}$, revised to focus on vegetable preparation and parent behavioral strategies, on child vegetable intake, liking, and variety with outcomes measured at baseline, immediate post-, 6-, and 12 months post-course. Other dietary, environmental, and psychosocial outcomes based on the cooking intervention were also evaluated.

Description: A series of six weekly Cooking Matters for Families lessons focused on vegetable procurement and preparation was implemented for low-income parents (96\% women, 60\% high school education, $16 \%$ white) and youth (9-12 years, $62 \%$ girls, $12 \%$ white, $50 \%$ overweight/obese) between 2014-2016 in community-based settings. Lessons lasting about two hours were delivered by a chef and extension nutrition educator.

Evaluation: Interim analyses are underway to compare baseline and immediate post-course outcomes for a combined group of intervention and control families $(n=89)$. Results will be reported for parent and child vegetable intake, parent and child liking and home availability for 18 vegetables used in the cooking course and 19 others, parent confidence in preparing vegetables and in using 10 vegetable cooking methods, overall parent and child cooking confidence, parent healthy food preparation and food resource management practices, and child interest in cooking. When twelve month post-course data are available from all families, final analysis will also be completed to compare all outcomes between the intervention and control groups. Conclusions and Implications: The results will be used to inform further dissemination of the Cooking Matters for Families program revised to focus on vegetable preparation and parent behavioral strategies for selected outcomes.

Funding: 2012-68001-19631

\section{NP23 Barriers to Healthy Eating and Physical Activity for the Development of Nutrition Materials for Puerto Rican Children}

Maria Rodriguez,PhD, mariadc.rodriguez@upr.edu, University of Puerto Rico-Mayagũez, 259 Alfonso Valdés Cobián Ave., Mayagũez, Puerto Rico 681;

Nancy Correa, PhD, LDN, RDN, University of Puerto RicoRío Piedras; Robinson Rodriguez, PhD, University of Puerto Rico-Mayaguez 